\title{
Simplified Application of Quadrupolar Excitation in Fourier Transform Ion Cyclotron Resonance Mass Spectrometry
}

\author{
C. L. Hendrickson, J. J. Drader, and D. A. Laude, Jr. \\ Department of Chemistry and Biochemistry, The University of Texas at Austin, Austin, Texas, USA
}

A new method for application of quadrupolar excitation to the trapped ion cell of a Fourier transform ion cyclotron resonance (FTICR) mass spectrometer is presented. Quadrupolar excitation is conventionally applied to the two pairs of opposed electrodes that normally perform the excitation and detection functions in the FTICR experiment. Symmetry arguments and numerically calculated isopotential contours within the trapped ion cell lead to the conclusion that quadrupolar excitation can be applied to a single pair of opposed side electrodes. Examples of effective quadrupolar axialization via this method include a sevenfold signal-to-noise enhancement derived from 50 remeasurements of a single population of trapped bovine insulin ions and the selective isolation of a single charge state of horse heart myoglobin after an initial measurement that revealed the presence of 14 charge states. (J Am Soc Mass Spectrom 1995, 6, 448-452)

$\mathrm{O}$ ne of the most promising recent advances in Fourier transform ion cyclotron resonance (FTICR) mass spectrometry is the development of quadrupolar excitation (also known as quadrupolar axialization) for ion cooling and centering [1-14]. A resonant electric azimuthal quadrupolar field is used to reduce ion magnetron radii through coupling of the cyclotron and magnetron motions [1-3] in the presence of a suitable collision gas. The defeat of this major ion cloud expansion and loss process has allowed researchers to achieve remarkable improvements in ion confinement time [4, 5], resolution [6-8], selectivity $[5,8-10]$, remeasurement efficiency [11], and collisioninduced dissociation (CID) efficiency [12].

Typically quadrupolar excitation is applied to the two pairs of opposed electrodes that make up the side plates of the trapped ion cell. A small potential that oscillates at the unperturbed ion cyclotron frequency is applied to one pair of opposed side plates and a second waveform of the same amplitude and frequency, but $180^{\circ}$ out of phase with the first waveform, is applied to the remaining pair of side electrodes. This arrangement creates a two-dimensional quadrupolar field in the $x-y$ (azimuthal) plane of the trapped ion cell. During conventional FTICR operation one pair of side electrodes is used for dipolar cyclotron excitation and the other pair is used for differential detection. Thus, quadrupolar excitation and dipolar excitation

Address reprint requests to David A. Laude, Jr., The University of Texas at Austin, Austin, TX 78712. require different electrical connections to the side electrodes of the cell and to that extent are incompatible.

To date two solutions to this incompatibility have been implemented. The first, developed by Marshall's group [4], requires two trapped ion cells that share a common trap plate (referred to as the dual cell). One cell (called the source cell) is wired for quadrupolar excitation and the other (called the analyzer cell) is wired for dipolar excitation and detection. In general the experiments involve ion trapping and quadrupolar axialization in the source cell, ion transfer to the analyzer cell through a small orifice in the common trap plate, and dipolar excitation and detection in this suitably wired cell. Although this method has been highly successful, it does require two cells and is somewhat inflexible. Once the trapped ions have been transferred to the analyzer cell, the quadrupolar axialization capability is lost and only a limited number of ion detection events are possible.

The second solution is to rapidly switch the electrical connections of the cell side plates from dipolar mode to quadrupolar mode by using a relay inserted between the cell electrodes and the instrument electronics. This implementation of quadrupolar axialization was first used by Speir et al. [11] to achieve highly efficient remeasurement in a cubic cell. Advantages of this techrique include the need for only one ion trap and the flexibility to switch between modes of operation as desired. However, the method does require additional hardware that is not commercially available, and insertion of the switching relay into the instrument electronics can impair their performance. 
For example, the additional capacitive load of the switching circuit may exceed the capability of the excitation amplifier and introduces excess noise picked up by the detection preamplifier.

Consideration of the excitation field symmetry required to facilitate quadrupolar excitation reveals that the excitation waveform can be applied to only one opposed pair of side electrodes. In this work, the successful application of quadrupolar excitation to only two of the side plates of the trapped ion cell is demonstrated. This method of operation simplifies the quadrupolar axialization procedure and reduces noise in the detection circuitry by removing the detection electrodes from the cooling process. SIMIONgenerated [15] isopotential contours show that the shape of the isopotential contours is nearly identical in the comparison of excitation on two and four plates. Examples of the capability of this new method of operation include remeasurement of a single ion population and charge state isolation through selective cooling of a single protein charge state.

\section{Experimental}

An electrospray ionization mass spectrometer was used to collect the experimental data and is described elsewhere $[16,17]$. Briefly, electrospray ions are formed at atmospheric pressure in a Chait-type source [18] positioned in the bore of a 3.0-T superconducting magnet. Differential pumping across a series of concentric vacuum chambers reduces the system pressure to $10^{-9}$ torr at a cylindrical open trapped ion cell [19] positioned $15 \mathrm{~cm}$ away in the center of the magnet. Ion kinetic energy is determined by a supersonic gas expansion across the heated metal capillary and $250-\mu \mathrm{m}$ diameter skimmer and by the potential applied to the skimmer, which is typically in the 5-15-V range. Typical trap potentials used to capture ions are between 5 and $10 \mathrm{~V}$. An Odyssey data station (Waters Extrel FTMS, Madison, WI) was used for all instrument control, data acquisition, and spectral presentation. Ions were excited by using a $70-V_{p-p}$ chirp swept at 400 $\mathrm{Hz} / \mu \mathrm{s}$, and $16 \mathrm{~K}$ data points were digitized by using a $600-\mathrm{kHz}$ bandwidth. The resulting transients were baseline corrected and zero padded prior to magnitude-mode Fourier transformation. Bovine insulin (MW 5735) and horse myoglobin (MW 16951) were used as received from Sigma Chemical Co. (St. Louis, MO).

Quadrupolar excitation is applied to the cell by using a previously described switching box [11]. The circuit consists of a four pole, double throw relay that is triggered by a 5-V TTL pulse. When the relay switches are active, the electrical connections at the cell facilitate the application of an azimuthal quadrupolar electric field. The collision gas is admitted to the cell region from a differentially pumped reservoir, which for this work was maintained at a pressure of 1 torr. This allowed the pulse valve to remain open during the entire axialization event (1-5 s), which thus simulated a static pressure [7] and improved performance. The optimum quadrupolar excitation voltage for all experiments was found to be between 0.2 and $2 V_{p-p}$. Typical cooling delays ranged from 1 to $5 \mathrm{~s}$.

The difference between conventionally applied quadrupolar axialization and the method described here is shown in Figure 1. The usual electrode connections are shown in Figure 1a. In this case the two electrodes used for dipolar excitation are switched to one output of the excitation amplifier and the two electrodes used for dipolar detection are switched to the remaining output, which is $180^{\circ}$ out of phase with respect to the first output. The application of quadrupolar excitation to only two electrodes is shown in Figure $1 \mathrm{~b}$. The two detection electrodes are simply wired directly to the detection preamplifier (or can be switched for other purposes).

\section{Results and Discussion}

At first glance the symmetry necessary to form an azimuthal quadrupolar field may lead to the conclusion that voltages must be applied to all four side plates of a typical trapped ion cell. However, all that really is required is the presence of the four electrodes. Just as in the simplest operational mode of the quadrupole ion trap, in which the end caps are held at earth ground and voltage is applied to the ring elec-
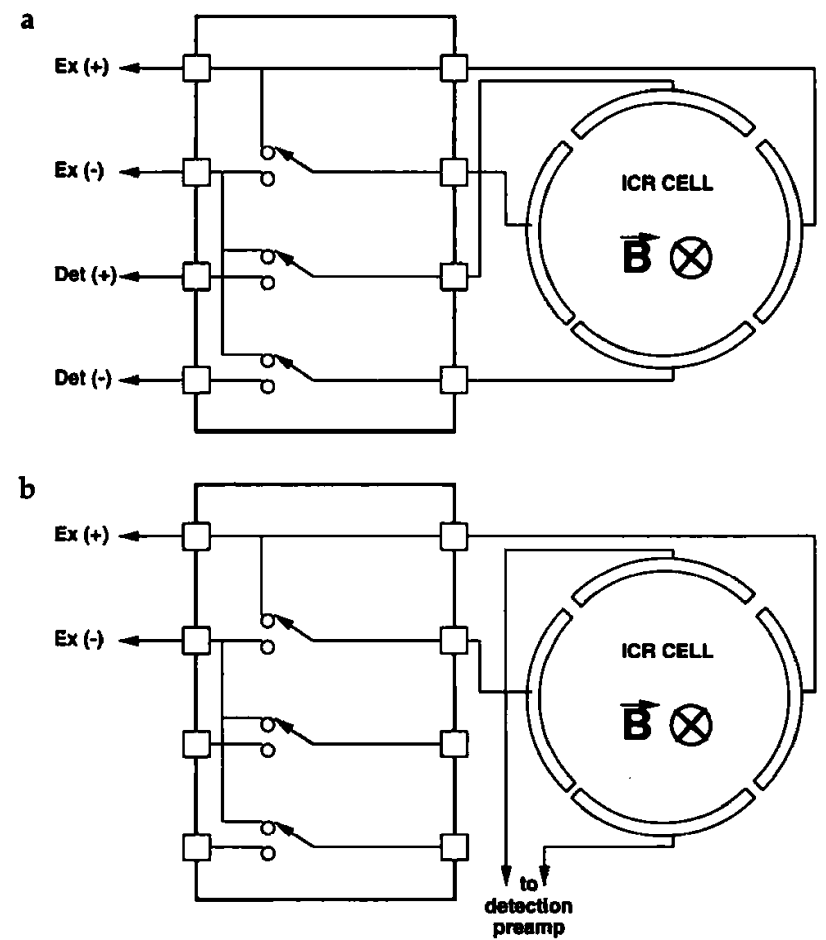

Figure 1. Schematic diagram of the electrical connections to the trapped ion cell when (a) quadrupolar excitation is applied conventionally and (b) when quadrupolar excitation is applied to only one pair of cell electrodes. 
trode [20], an excitation signal need be applied only to one pair of side electrodes, while the other pair is held at ground potential to shape the azimuthal quadrupolar field. Evidence in support of this argument is shown in Figure 2 in the form of SIMION-generated isopotential contours for a trapped ion cell of square cross section. In Figure 2a conventionally applied quadrupolar excitation is depicted in which $1 \mathrm{~V}$ is applied to one set of opposed electrodes and $-1 \mathrm{~V}$ is applied to the remaining set. The familiar shape of the contours can be immediately recognized. Modeled in Figure $2 b$ is the same cell but with one set of opposed electrodes at $2 \mathrm{~V}$ and the other pair at ground $(2 \mathrm{~V}$ is applied instead of $1 \mathrm{~V}$ so that the potential difference between adjacent electrodes remains constant). The shape of the contours, as expected, is similar to those shown in Figure 2a. It should be noted that the square cross section geometry was chosen only for the convenience of modeling flat electrodes and that the argument holds for hyperbolic and cylindrical electrodes as well. In fact, the experimental data to be shown in support of the theory were collected in an elongated open cell of circular cross section.

Although not apparent in Figure 2, the cell potential is not identical in the two cases. For example, when excitation is applied conventionally (as shown in Figure 1a), the potential at the center of the cell remains constant. When excitation is applied to only two plates (as in Figure 1b) the potential at the cell center oscil-
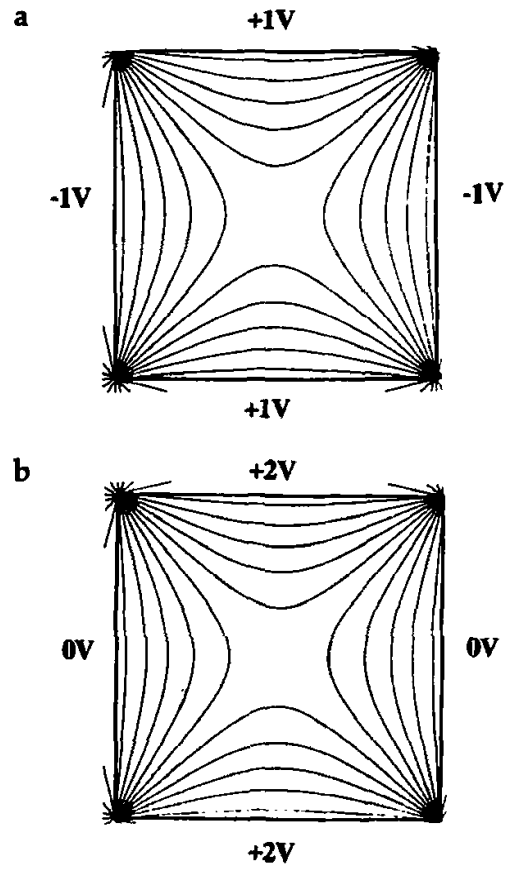

Figure 2. SIMION-generated isopotential contours for a cell of square cross section when (a) quadrupolar excitation is applied conventionally and (b) when quadrupolar excitation is applied to only one pair of cell electrodes. In both cases the isopotential contours are spaced every $0.2 \mathrm{~V}$. lates. The result is an additional axial resonance not present in conventional quadrupolar excitation. However, even if this resonance is excited (for instance, during broadband axialization), the high pressure used during the quadrupolar excitation event would prohibit any significant increase in motional amplitude and subsequent ion loss. In fact, there are additional resonarces present in conventional quadrupolar excitation $[3,21]$ that do not impair broadband performance for precisely this reason.

One of the most rigorous tests of the cooling process is to remeasure the same ion population many times [22-24]. Analysis of the signal-to-noise ratio $(\mathrm{S} / \mathrm{N})$ enhancement then can be related to the efficiency of axialization for a given time period, dipolar excitation radius, and number of remeasurements. Shown in Figure 3 are comparison mass spectra of the +5 charge state of electrosprayed bovine insulin ions. The single scan spectrum exhibits a $\mathrm{S} / \mathrm{N}$ of about 10 . By remeasuring that same ion population 50 times, a $\mathrm{S} / \mathrm{N}$ of 70 is achieved. The $n^{1 / 2}$ improvement in signal-to-noise ratio indicates that the ion cloud has cooled completely to the center of the trapped ion cell after each measurement and that few, if any, ions were lost during the 5-min experiment.

a

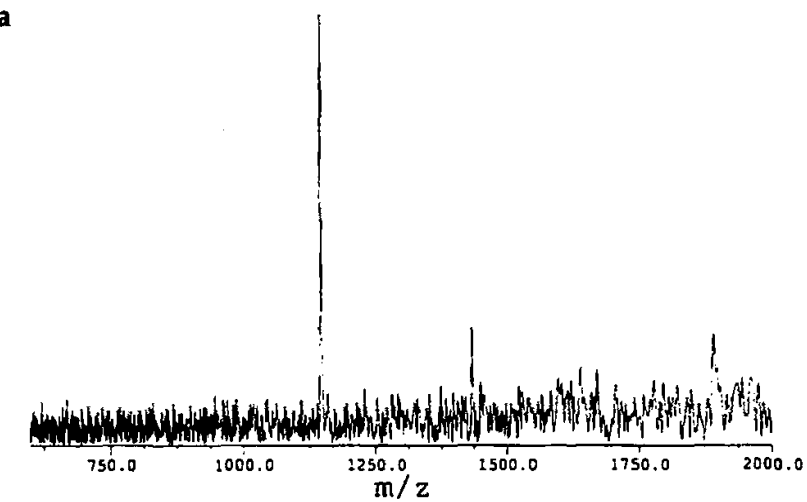

b

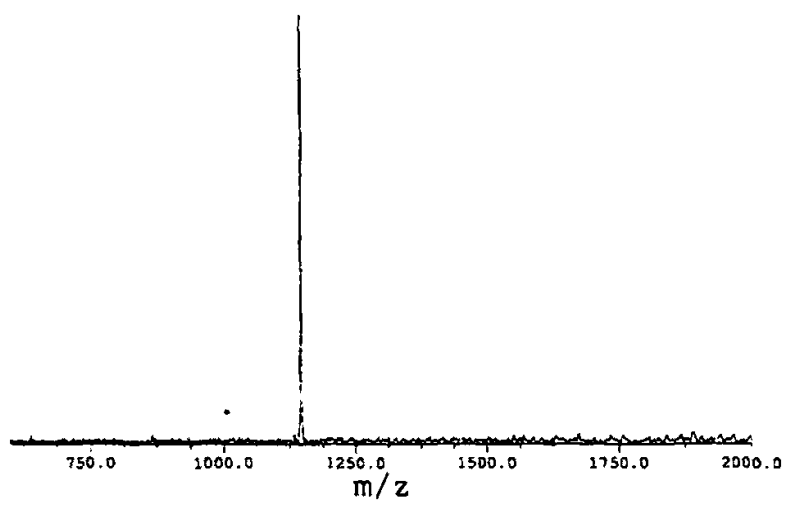

Figure 3. Comparison electrospray ionization FTICR mass spectra of the +5 charge state of bovine insulin. A sevenfold $S / N$ enhancement is observed for the comparison of (a) the spectrum obtained from the first scan with (b) the spectrum obtained from 50 remeasured scans. 
For the interconversion between cyclotron and magnetron motion to occur, the quadrupolar electric field must resonate at the cyclotron frequency of the chosen ion. This mass selectivity easily can be used to isolate a single protein charge state. Shown in Figure 4 is the isolation of a single charge state of horse myoglobin. The experiment was performed by measuring a single ion population twice. The first observation is shown in Figure 4a. It is evident that 14 charge states of myoglobin have been trapped and detected. Following detection the pulse valve was opened for $5 \mathrm{~s}$ to raise the pressure in the cell to $10^{-5}$ torr, while quadrupolar excitation was applied at the frequency of the +18 charge state. The spectrum shown in Figure $4 \mathrm{~b}$ then was acquired under the same excitation conditions used for the first scan. The selective nature indicative of quadrupolar excitation is evident. The +18 charge state been isolated from $a+18$ adduct species as well as from the other charge states.

The increased flexibility afforded by using only two side plates offers many potential advantages over the conventional method. As previously shown [25] removal of the switching relay from the detection elec-

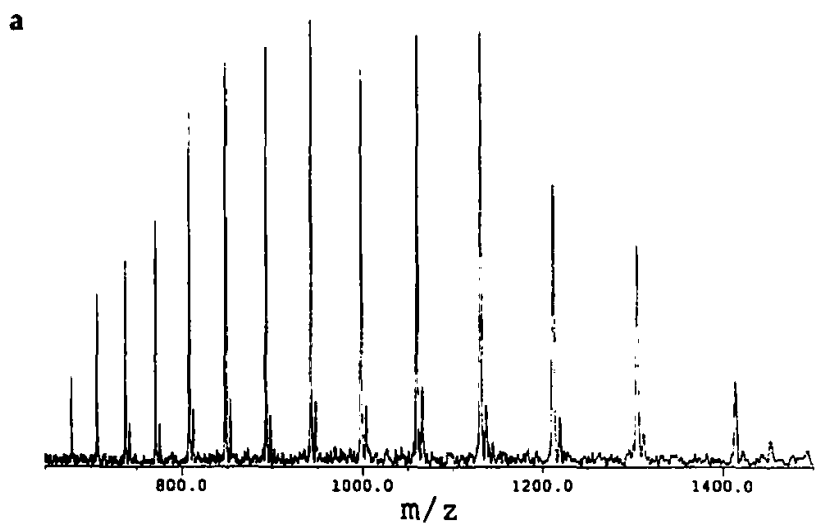

b

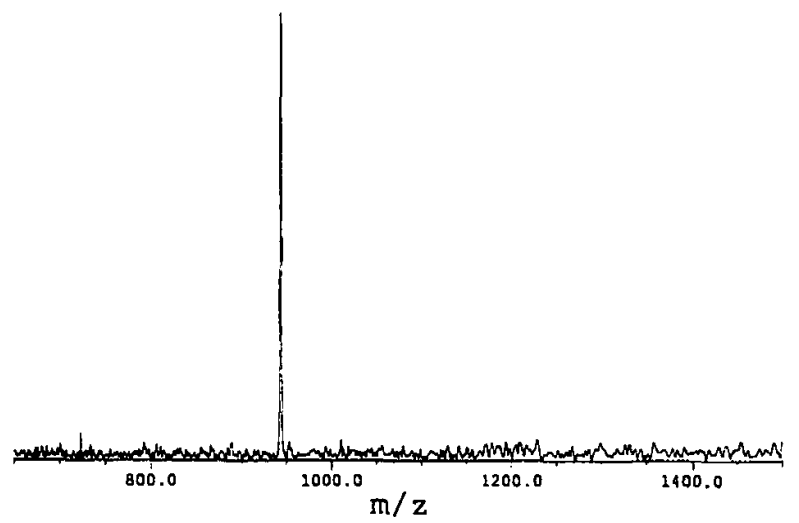

Figure 4. Electrospray ionization FTICR mass spectra of a single population of horse myoglobin ions (a) before and (b) after application of selective quadrupolar axialization to the +18 charge state. A $500-m V_{p-p}$ quadrupolar excitation signal was applied on the two excitation plates for $5 \mathrm{~s}$ while the pressure in the cell region was maintained at $1 \times 10^{-5}$ torr. tronics eliminates excess noise. In fact, the switching relay can be eliminated entirely by employing a center-tapped transformer. In this configuration the dipolar excitation waveform is applied to the input of the transformer and the quadrupolar excitation signal is applied to the center tap of the output, which results in the proper phase relationship for both signals. Alternatively, the detection electrodes could be switched to apply an additional excitation simultaneously with the quadrupolar excitation [12].

\section{Conclusions}

Single-frequency quadrupolar excitation can be successfully applied to only two side plates of a FTICR trapped ion cell with no compromise in axialization efficiency. From symmetry arguments and SIMIONgenerated isopotential contours it can be seen that excitation on only two plates forms the same azimuthal quadrupolar field as the standard four plate excitation. Examples of quadrupolar axialization presented include efficient ion remeasurement and charge state selection. Excitation on only two plates can free the detection circuitry from the complicating effects of a switching relay or, if desired, more elaborate experiments can be performed.

\section{Acknowledgment}

The authors thank A. G. Marshall and M. W. Senko for helpful discussions. This work was supported by the Welch Foundation, the Texas Advanced Research Program, the National Institutes of Health, and the National Science Foundation.

\section{References}

1. Savard, G.; Becker, St.; Bollen, G.; Kluge, H.-J.; Moore, R. B.; Otto, Th.; Schweikhard, L.; Stolzenberg, H.; Wiess, U. Phys. Lett. A 1991, 158, 247.

2. Becker, St.; Bollen, G.; Kern, F.; Kluge, H.-J.; Moore, R. B.; Savard, G.; Schweikhard, L.; Stolzenberg, H. Int. J. Mass Spectrom. Ion Processes 1990, 99, 53.

3. Bollen, G.; Moore, R. B.; Savard, G.; Stolzenberg, H. J. Appl. Phys. 1990, 68, 4355.

4. Schweikhard, L.; Guan, S.; Marshall, A. G. Int. J. Mass Spectrom. Ion Processes 1992, 120, 71.

5. Bruce, J. E.; Anderson, G. A.; Hofstadler, S. A.; Van Orden, S. L.; Sherman, M. S.; Rockwood, A. L.; Smith, R. D. Rapid Commun. Mass Spectrom. 1993, 7, 914.

6. Guan, S.; Wahl, M. C.; Wood, T. D.; Marshall, A. G. Anal. Chem. 1993, 65, 1753.

7. Guan, S.; Marshall, A. G. Rapid Commun. Mass Spectrom. 1993, $7,857$.

8. Wahl, M. C.; Kim, H. S.; Wood, T. D.; Guan, S.; Marshall, A. G. Annl. Chem. 1993, 65, 3669.

9. Guan, S.; Marshall, A. G. I. Chem. Phys. 1993, 98, 4468.

10. Guan, S.; Wahl, M. C.; Marshall, A. G. J. Chem. Phys. 1994, $100,6137$.

11. Speir, J. P.; Gorman, G. S.; Pitsenberger, C. C.; Turner, C. A.; Wang, P. P.; Amster, I. J. Anal. Chem. 1993, 65, 1746. 
12. Guan, S.; Marshall, A. G.; Wahl, M. C. Anal. Chem. 1994, 66 1363.

13. Guan, S.; Xiang, X.; Marshall, A. G. Int. J. Mass Spectrom. Ion Processes 1993, 124, 53.

14. Chen, R.; Guan, S.; Marshall, A. G. J. Chem. Phys. 1994, 100, 2258.

15. Dahl, D. A.; Delmore, J. E. SIMION 4.0, Idaho National Engineering Laboratory, Idaho Falls, ID, 1988.

16. Hofstadler, S. A.; Laude, D. A., Jr. J. Am. Soc. Mass Spectrom. $1992,3,615$.

17. Campbell, V. L.; Guan, Z.; Laude, D. A., Jr. J. Am. Soc. Mass Spectrom. 1994, 5, 221.

18. Katta, V,; Chowdhury, S. K.; Chait, B. T. I. Am. Chem. Soc. $1990,112,5348$.
19. Beu, S. C.; Laude, D. A., Jr. Int. J. Mass Spectrom. Ion Processes 1992, 112, 215.

20. March, R. E.; Hughes, R. J. Quadrupole Storage Mass Spectrometry; Wiley: New York, 1991.

21. Schweikhard, L.; Marshall, A. G. J. Amt. Soc. Mass Spectrom. 1993, 4, 433.

22. Williams, E. R.; Henry, K. D.; McLafferty, F. W. I. Am. Chem. Soc. 1990, 112, 6157.

23. Guan, Z.; Hofstadler, S. A.; Laude, D. A., Jr. Anal. Chem. 1993, 65, 1588.

24. Campbell, V. L.; Guan, Z.; Vartanian, V. H.; Laude, D. A., Jr. Anal. Chem., 1995, 67, 420.

25. Hendrickson, C. L.; Laude, D. A., Jr. Anal. Chem., to appear. 